\title{
Optimization of Flapping Airfoils for Maximum Thrust and Propulsive Efficiency
}

\author{
Ismail H. Tuncer* and Mustafa Kaya ${ }^{\dagger}$ \\ Middle East Technical University, 06531 Ankara, Turkey
}

\begin{abstract}
The thrust and/or propulsive efficiency of a single flapping airfoil is maximized by using a numerical optimization method based on the steepest ascent. The flapping motion of the airfoil is described by a combined sinusoidal plunge and pitching motion. Optimization parameters are taken to be the amplitudes of the plunge and pitching motions and the phase shift between them at a fixed flapping frequency. Two-dimensional, unsteady, low-speed, laminar, and turbulent flows are computed by using a Navier-Stokes solver on moving overset grids. Computations are performed in parallel in a computer cluster. The optimization data show that high thrust values may be obtained at the expense of propulsive efficiency. For a high propulsive efficiency, the effective angle of attack of the airfoil is reduced, and large-scale vortex formations at the leading edge are prevented.
\end{abstract}

\section{Nomenclature}

$a_{\infty}=$ freestream speed of sound

$C_{t}=$ average thrust coefficient

$c=$ airfoil chord length

$h=$ plunge position

$h_{o}=$ plunge amplitude nondimensionalized with $c$

$k=$ reduced frequency, $\omega c / U_{\infty}$

$R e=$ Reynolds number based on the chord length

$T=$ period of a flapping motion

$t=$ time nondimensionalized with $a_{\infty} / c$

$U_{\infty}=$ freestream velocity

$\alpha=$ pitch angle

$\alpha_{o}=$ pitch amplitude

$\eta \quad=$ propulsive efficiency

$\omega=$ angular frequency

\section{Introduction}

B ASED on observations of flying birds and insects and swimming fish in nature, flapping-wing propulsion has already been recognized to be more efficient than conventional propellers for very smallscale vehicles with wing spans of $15 \mathrm{~cm}$ or less, so-called micro-air vehicles (MAVs). Because the primary mission for MAVs is surveillance, they are desired to have good maneuverability and sustained flights with flight speeds of 30-60 km/h. The current interest in the research and development community is, therefore, to find the most energy-efficient airfoil adaptation and wing motion technologies capable of providing the required aerodynamic performance for a MAV flight.

Recent experimental and computational studies investigated the kinematics, dynamics, and flow characteristics of flapping wings and shed some light on the lift, drag, and propulsive power considerations. ${ }^{1,2}$ Water-tunnel flow visualization experiments on flapping airfoils conducted by Lai and Platzer ${ }^{3}$ and Jones et al. ${ }^{4}$ provide a considerable amount of information on the wake char-

Presented as Paper 2003-0420 at the AIAA 41st Aerospace Sciences Meeting, Reno, NV, 6-9 January 2003; received 21 May 2003; revision received 23 June 2004; accepted for publication 28 June 2005. Copyright (C) 2005 by Ismail H. Tuncer and Mustafa Kaya. Published by the American Institute of Aeronautics and Astronautics, Inc., with permission. Copies of this paper may be made for personal or internal use, on condition that the copier pay the $\$ 10.00$ per-copy fee to the Copyright Clearance Center, Inc., 222 Rosewood Drive, Danvers, MA 01923; include the code 0001-1452/05 \$10.00 in correspondence with the CCC.

*Professor, Department of Aerospace Engineering; tuncer@ae.metu. edu.tr.

Graduate Research Assistant, Department of Aerospace Engineering; mkaya@ae.metu.edu.tr. acteristics of thrust-producing flapping airfoils. In their experiments, Anderson et al. ${ }^{5}$ observed that the phase angle between pitch and plunge oscillations plays a significant role in maximizing the propulsive efficiency. Navier-Stokes computations performed by Tuncer and Platzer, ${ }^{6,7}$ Tuncer et al., ${ }^{8,9}$ Isogai et al., ${ }^{10}$ and Isogai and Shinmoto ${ }^{11}$ explore the effect of flow separation on the thrust generation and the propulsive efficiency of a single flapping airfoil in combined pitch and plunge oscillations.

Jones and Platzer ${ }^{12}$ and Jones et al. ${ }^{13}$ recently demonstrated a radio-controlled MAV propelled by flapping wings in a biplane configuration (Fig. 1). The experimental and numerical studies by Jones and Platzer, ${ }^{12}$ Jones et al. ${ }^{14,15}$ and Platzer and Jones ${ }^{16}$ on flappingwing propellers points at the gap between numerical results and the actual flight conditions with high-frequency flapping wings. They also observed that the performance of flapping airfoils may be enhanced or limited by the onset of dynamic stall, and large-scale flow separation.

In our earlier study, ${ }^{9}$ the average thrust coefficient of a NACA0012 airfoil flapping in plunge was obtained for a range of reduced frequencies and amplitudes of the flapping motion (Fig. 2). As can be seen, the thrust coefficient varies significantly with the frequency and amplitude of the flapping motion. The computational and experimental findings show that thrust generation and propulsive efficiency of flapping airfoils are closely related to the flapping motion and flow parameters, such as the flapping frequency, the amplitude of the pitch and plunge motions, the phase shift between them, air speed, and turbulence. It is apparent that to maximize the thrust and/or propulsive efficiency of a flapping airfoil, an optimization of all of these variables is needed.

In this study, the average thrust and/or the propulsive efficiency of a flapping airfoil undergoing a combined plunge and pitch motion (Fig. 3) is maximized by using a gradient-based optimization process. The optimization variables are taken to be the pitch and plunge amplitudes $h_{o}$ and $\alpha_{o}$ and the phase shift between the pitch and plunge motions $\phi$. The gradient of the objective function is evaluated numerically by perturbing the optimization variables and computing the unsteady flowfield for a few periods of the flapping motion until a periodic flowfield is established. The flowfield around a flapping airfoil is discretized by using moving overset grids. The unsteady flow solutions for the evaluation of the gradient vector are computed in parallel in a computer cluster.

\section{Numerical Method}

Two-dimensional unsteady viscous flows around a flapping airfoil are computed by solving the Navier-Stokes equations on moving overset grids. The flow variables at the intergrid boundaries of overset subgrids are interpolated from the donor subgrid. Computations on each subgrid are performed in parallel. Parallel virtual machine 


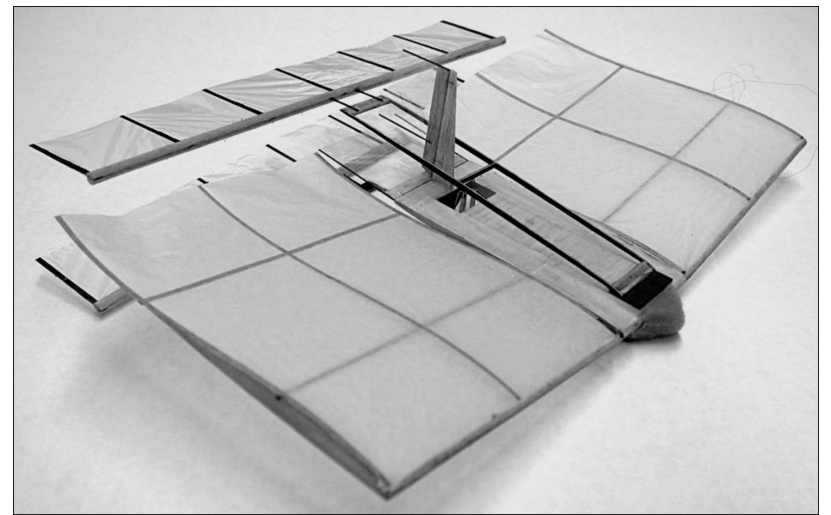

Fig. 1 MAV with flapping wings. ${ }^{13}$

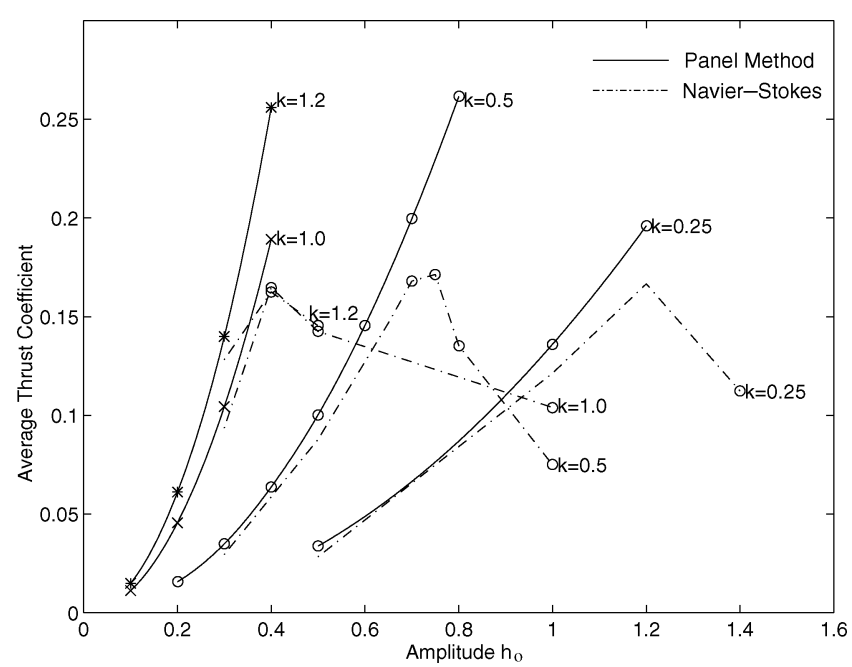

Fig. 2 Variation of thrust coefficient for a flapping airfoil in plunge. ${ }^{9}$

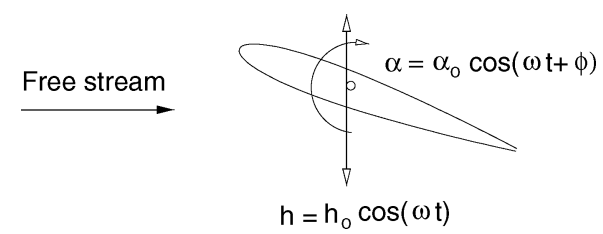

Fig. 3 Flapping motion of an airfoil in combined plunge and pitch.

(PVM) message-passing library routines are used in the parallel solution algorithm. ${ }^{17}$ The unsteady flow solutions are analyzed in terms of average thrust coefficient and propulsive efficiency values and unsteady particle traces.

\section{Navier-Stokes Solver}

The strong conservation-law form of the two-dimensional, thinlayer, Reynolds-averaged Navier-Stokes equations is solved on each subgrid. The governing equations in a curvilinear coordinate system $(\xi, \zeta)$ are given as follows:

$$
\partial_{t} \hat{\boldsymbol{Q}}+\partial_{\xi} \hat{\boldsymbol{F}}+\partial_{\zeta} \hat{\boldsymbol{G}}=R e^{-1} \partial_{\zeta} \hat{\boldsymbol{S}}
$$

where $\hat{\boldsymbol{Q}}$ is the vector of conservative variables, $\hat{\boldsymbol{G}}$ are the convective flux vectors, and $\hat{\boldsymbol{S}}$ is the thin-layer approximation of the viscous fluxes in the $\zeta$ direction normal to the airfoil surface. ${ }^{6}$ The convective fluxes are evaluated by using the third-order-accurate Osher's upwind-biased flux difference splitting scheme. In turbulent flow computations, the Baldwin-Lomax turbulence model is used.

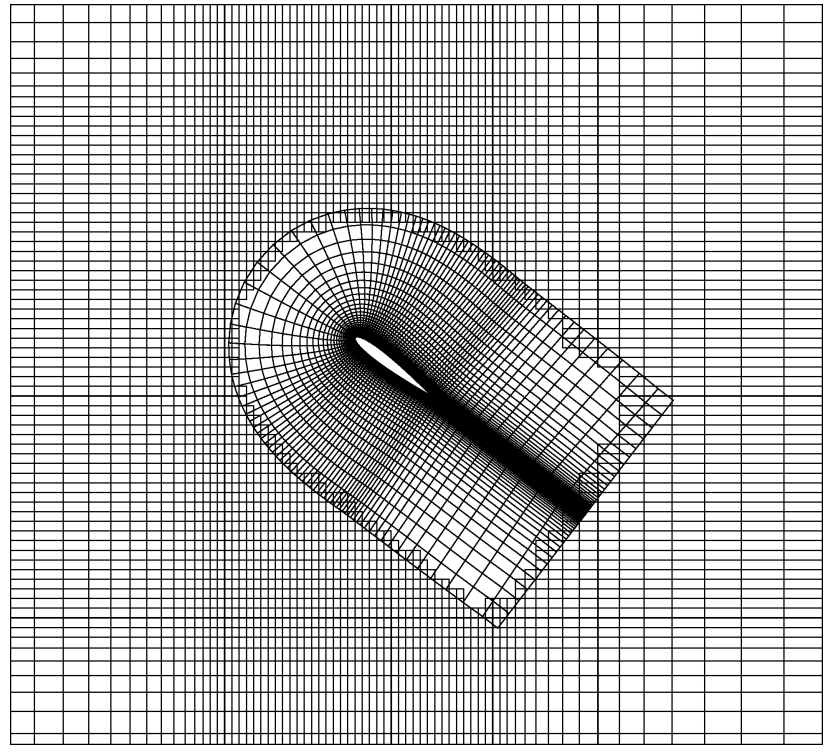

Fig. 4 Overset grid system.

The discretized equations are solved by an approximately factored, implicit algorithm. The holes in the background grid formed by the airfoil grid are excluded from the computations by an "i”-blanking algorithm. ${ }^{17}$

\section{Computational Domain}

The computational domain is discretized with overset grids. A Ctype grid around the airfoil is overset onto a Cartesian background grid (Fig. 4). In our earlier study, ${ }^{9}$ grid-independent solutions on overset grid systems were already established. The flapping motion of the airfoil is imposed by moving the airfoil and the grid around it on the background grid. The flapping motion of the airfoil in combined plunge $h$ and pitch $\alpha$ is specified by

$$
h=-h_{o} \cos (\omega t), \quad \alpha=-\alpha_{o} \cos (\omega t+\phi)
$$

where the angular frequency $\omega$ is given in terms of the reduced frequency, $k=\omega c / U_{\infty} ; \phi$ is the phase shift between plunge and pitching motions. The pitching motion is about the midchord location.

\section{Intergrid Boundary Conditions}

At the intergrid boundaries formed by the overset grids, the conservative flow variables are interpolated in each timestep of the unsteady solution. Intergrid boundary points are first localized in a triangular stencil in the donor grid by a directional search algorithm. The localization process incidentally provides the interpolation weights to interpolate the flow variables within the triangular stencil. $^{17}$

\section{Optimization}

The objective function is taken as a linear combination of the average thrust coefficient $C_{t}$ and the propulsive efficiency $\eta$ over a flapping period:

$$
\begin{gathered}
O\left(C_{t}, \eta\right)=(1-\beta) \frac{C_{t}}{C_{t}+\varepsilon\left|\nabla C_{t} \cdot \boldsymbol{D}\right|}+\beta \frac{\eta}{\eta+\varepsilon|\nabla \eta \cdot \boldsymbol{D}|} \\
C_{t}=-\frac{1}{T} \int_{t}^{t+T} C_{d} \mathrm{~d} t \\
\eta=C_{t} U_{\infty} / \frac{1}{T} \int_{t}^{t+T} \int_{S} p(\boldsymbol{V} \cdot d \boldsymbol{A}) \mathrm{d} t
\end{gathered}
$$

The denominator in the efficiency expression accounts for the average work required to maintain the flapping motion; $\varepsilon$ denotes the 
optimization step size. Note that $\beta=0$ sets the objective function to a normalized thrust coefficient.

The optimization process is based on following the direction of the steepest ascent of the objective function $O$. The direction of the steepest ascent is given by the gradient vector of the objective function:

$$
\boldsymbol{\nabla} O(\boldsymbol{V})=\frac{\partial O}{\partial V_{1}} \boldsymbol{v}_{1}+\frac{\partial O}{\partial V_{2}} \boldsymbol{v}_{2}+\cdots
$$

where $V_{i}$ are the optimization variables and $v_{i}$ are the corresponding unit vectors in the variable space.

The components of the gradient vector is then evaluated numerically by computing the objective function for a perturbation of all of the optimization variables one at a time. Note that the evaluation of these vector components requires an unsteady flow solution over a few periods of the flapping motion until a periodic flow behavior is reached. Once the unit vector in the steepest ascent direction is evaluated by

$$
\boldsymbol{D}=\boldsymbol{\nabla} O /|\nabla O|
$$

a small step in the steepest ascent direction, $\Delta \boldsymbol{V}=\varepsilon \boldsymbol{D}$, is taken until a local maximum is reached. Reference 18 suggests that the step size $\varepsilon$ should be such that $|\nabla O(\boldsymbol{V}+\varepsilon \boldsymbol{D})|^{2}$ is minimum. That is,

$$
\frac{\partial\left(|\boldsymbol{\nabla} O(\boldsymbol{V}+\varepsilon \boldsymbol{D})|^{2}\right)}{\partial \varepsilon}=0
$$

An approximate solution for $\varepsilon$ based on the second-order Taylor series expansion is given by ${ }^{18}$

$$
\varepsilon=-\frac{\nabla O \cdot(\nabla \nabla O) \cdot \boldsymbol{D}}{\boldsymbol{D} \cdot(\nabla \nabla O) \cdot(\nabla \nabla O) \cdot \boldsymbol{D}}
$$

where $\nabla \nabla O$ is a symmetric tensor known as the Hessian. The vector $(\nabla \nabla O) \cdot \boldsymbol{D}$ points at a direction where the gradient of the objective function becomes zero based on the quadratic expansion. The Hessian involves the second derivatives of the objective function with respect to optimization variables, $\partial^{2} O / \partial V_{i} \partial V_{j}$, and its computation is prohibitively expensive. However, to determine a proper step size, the following approximation is made based on the vector norms and the negative definiteness of the Hessian:

$$
\nabla O \cdot(\nabla \nabla O) \cdot \boldsymbol{D} \approx-|\nabla O \|(\nabla \nabla O) \cdot \boldsymbol{D}|
$$

Substitution into Eq. (1) then yields

$$
\varepsilon \approx|\nabla O| /|(\nabla \nabla O) \cdot D|
$$

$|(\nabla \nabla O) \cdot \boldsymbol{D}|$ is now evaluated by a backward finite difference approximation:

$$
|(\nabla \nabla O) \cdot \boldsymbol{D}| \approx\left|\nabla O\left(\boldsymbol{V}^{n}\right)-\nabla O\left(\boldsymbol{V}^{n-1}\right)\right| / \varepsilon^{n-1}
$$

\section{Parallel Computation}

A coarse parallel algorithm based on domain decomposition is implemented in a master-worker paradigm. ${ }^{19}$ The overset grid system is first decomposed into its subgrids, and the solution on each subgrid is assigned to a separate processor in the computer cluster. In addition, the background grid may be partitioned to improve the static load balancing. Intergrid boundary conditions are exchanged among subgrid processes at each timestep of the unsteady solution. PVM (Ver. 3.4.4) library routines are used for interprocess communication. In the optimization process, unsteady flow solutions with perturbed optimization variables, which are required to determine the gradient vector of the objective function, are all computed in parallel. Computations are performed in a cluster of computers with dual Pentium-III processors operating on a Linux operating system.

\section{Results and Discussion}

In this study the flapping motion parameters of a NACA0012 airfoil oscillating in a combined plunge and pitch is optimized for maximum thrust production and/or propulsive efficiency. As a validation of the method developed, a pure plunge motion of a NACA0012 airfoil is considered first. The flow is fully turbulent. The objective function is the thrust coefficient, and the plunge amplitude $h_{o}$ is the only optimization variable. Optimization steps are given in Fig. 5 and compared with the parametric study presented in Fig. 2. As shown, the optimization algorithm works well. The plunge amplitude $h_{o}=0.72$, which maximizes the thrust at $C_{t}=0.18$, is approached rapidly.

In the following study, the reduced frequency of the oscillatory motion is fixed at $k=1$. In an earlier optimization study with airfoils oscillating only in plunge, ${ }^{20}$ the reduced frequency was taken as an optimization variable, and it was established that the thrust production increases with the flapping frequency in the $k=1-4$ range. The optimization variables are now taken to be the plunge and pitch amplitudes $\left(h_{o}, \alpha_{o}\right)$ and the phase shift between plunge and pitch motions $(\phi)$. Table 1 summarizes the optimization cases studied and the initial values of the optimization variables. All of the flows are computed at $R e=1 \times 10^{4}$ and $M=0.1$, and except for case 11 , they are all assumed laminar in accordance with the flying MAV model ${ }^{13}$ The parallel computations with eight processors take about 20-30 h of wall-clock time for a typical optimization case.

In case 1 , where $\beta=0$, the average thrust coefficient is maximized. The time variation of the unsteady drag (negative thrust) coefficient along a few optimization steps is shown in Fig. 6. After the optimization variables are incremented along the optimization steps, the unsteady flow computations are carried out for several periods of the flapping motion until a periodic behavior is obtained. The periodicity of the flow is checked by comparing the average thrust values for consecutive periods. The variation of the average thrust coefficient and the propulsive efficiency with respect to the optimization

Table 1 Optimization cases and starting conditions

\begin{tabular}{lccrc}
\hline \hline Case & $\beta$ & $h_{o}$ & $\alpha_{o}$ & $\phi$ \\
\hline 1 & 0.0 & 0.5 & 5 & 30 \\
2 & 0.5 & 0.5 & 5 & 30 \\
3 & 1.0 & 0.5 & 5 & 30 \\
4 & 0.0 & 0.5 & 25 & 60 \\
5 & 0.0 & 1.0 & 5 & 60 \\
6 & 0.0 & 1.0 & 25 & 90 \\
7 & 1.0 & 0.5 & 25 & 60 \\
8 & 1.0 & 1.0 & 5 & 60 \\
9 & 1.0 & 1.0 & 25 & 90 \\
$10(M=0.2)$ & 0.0 & 0.5 & 5 & 30 \\
11 (Turb.) & 0.0 & 0.5 & 5 & 30 \\
\hline \hline
\end{tabular}

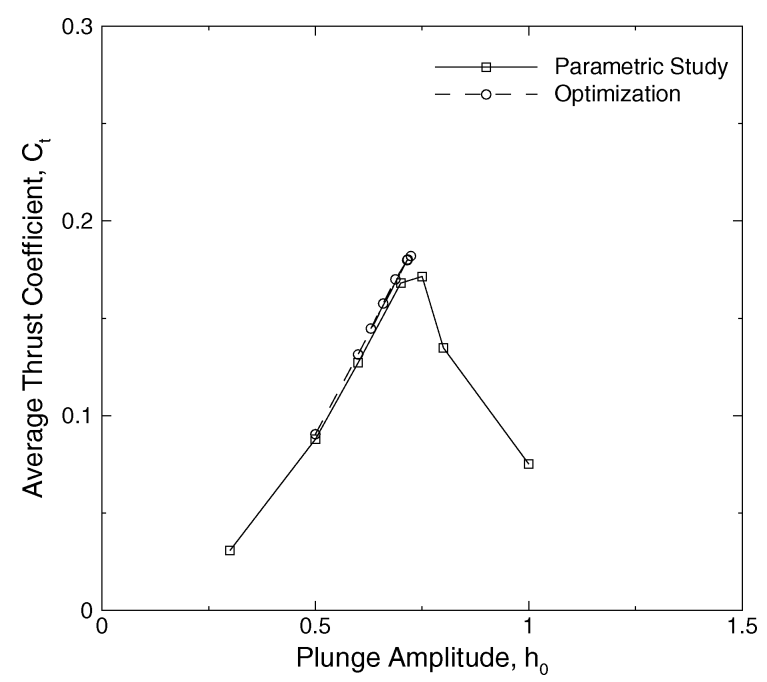

Fig. 5 Optimization of thrust coefficient at $M=0.3, R e=10^{6}, k=0.5$. 
variables are shown in Fig. 7. It is observed that as the optimization variables are incremented along the gradient vector of the objective function, the average thrust coefficient increases gradually. A maximum value of 1.41 is finally reached at $h_{o}=1.60, \alpha_{o}=23.5$, and $\phi=103.4 \mathrm{deg}$. The corresponding propulsive efficiency is $28.3 \%$.

All of the results of the optimization cases are given in Table 2. In case 2 , where $\beta=0.5$, the average thrust and the propulsive efficiency have equal weights in the objective function. As a result, the propulsive efficiency is improved at the expense of average thrust. It is observed that the higher efficiency is achieved at a lower plunge amplitude and a higher pitch amplitude. The phase shift slightly drops to $97.8 \mathrm{deg}$. In case 3, the propulsive efficiency is maximized at low pitch and plunge amplitudes with a corresponding very low thrust coefficient. It is well known that the propulsive efficiency and the thrust production of flapping airfoils are inversely proportional.

Table 2 Optimization results

\begin{tabular}{lccrcc}
\hline \hline Case & $h_{o}$ & $\alpha_{o}$ & \multicolumn{1}{c}{$\phi$} & $C_{t}$ & $\eta, \%$ \\
\hline 1 & 1.60 & 23.5 & 103.4 & 1.41 & 28.3 \\
2 & 1.36 & 29.6 & 97.8 & 1.08 & 44.1 \\
3 & 0.45 & 15.4 & 82.4 & 0.08 & 58.5 \\
4 & 1.73 & 23.8 & 100.7 & 1.44 & 25.4 \\
5 & 1.52 & 26.9 & 87.2 & 1.27 & 33.4 \\
6 & 1.55 & 28.6 & 94.9 & 1.45 & 35.9 \\
7 & 0.57 & 21.0 & 86.7 & 0.13 & 63.8 \\
8 & 0.60 & 22.8 & 86.1 & 0.13 & 64.8 \\
9 & 0.83 & 35.6 & 86.5 & 0.18 & 67.5 \\
10 & 1.53 & 20.0 & 94.9 & 0.95 & 23.2 \\
11 & 2.11 & 36.6 & 102.9 & 2.64 & 34.2 \\
\hline \hline
\end{tabular}

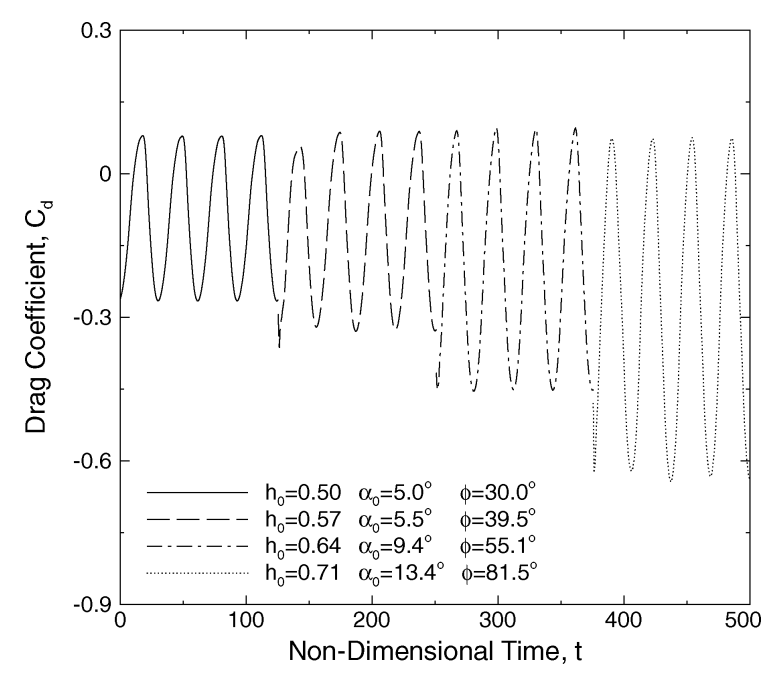

Fig. $6 C_{d}$ history along optimization steps, case 1: $\beta=0$.
The unsteady flowfields along the optimization steps are investigated with particle traces. The particles are emitted from a straight line in the vicinity of the leading edge of the airfoil and are convected in the flowfield with the local flow velocity. The vertical line from which the particles are emitted follows the leading-edge of the airfoil to capture the leading edge vortex formations in more detail. In Fig. 8, the instantaneous particle traces at the instant of maximum thrust (minimum drag) in a flapping period are given along the optimization steps of cases 1 and 3. The corresponding instantaneous pressure distributions on the airfoil are also given in Fig. 9. In both the particle traces and the leading-edge suction induced by the vortex shown in the pressure distributions, it is observed that in case 1, the leading-edge vortex formation is promoted along the optimization steps. The maximum instantaneous thrust occurs at about the mean amplitude position as the leading-edge vortex develops, just before the suction field at the leading edge collapses as the leading-edge vortex moves off the airfoil and is convected downstream, whereas in case 3, the leading-edge vortex, which is present initially, is first reduced in strength and finally disappears along the optimization steps. Instead, a weak trailing-edge vortex formation is observed. The unsteady flow now becomes more streamlined with the motion of the airfoil, and the propulsive efficiency is maximized at $58.5 \%$.

Figure 10 shows the optimized flowfield for maximum thrust in case 1 . The flowfield is observed to be highly vortical, with strong leading-edge vortices forming during the upstroke and the downstroke. The flowfield is periodic and antisymmetric along the upstroke and the downstroke.

Next, the optimization space is searched for other possible local maximums of the objective function for cases 1 and 3. It is implemented by initiating the optimization process from various initial conditions as given in Table 1 . The initial conditions and the optimized states at the end of the optimization processes are shown in Figs. 11 and 12 for $\beta=0$ and 1 , respectively. Figure 11 reveals that all of the optimization processes for $\beta=0$ converge about the same value of the average thrust coefficient $\left(C_{t} \approx 1.4\right)$ at about the same values of the optimization variables. It suggests that the global maximum of the objective function may have been found. On the other hand, although the optimization processes for maximizing propulsive efficiency converge about the same propulsive efficiency value ( $\eta \approx 65 \%$ ), the optimum states for $h_{o}$ and $\alpha_{o}$ show a considerably large variation (Fig. 12). It appears that the maximum flapping efficiency may be achieved in a range of $h_{o}$ and $\alpha_{o}$ values, where $\alpha_{o}$ increases as $h_{o}$ does in this range.

In case 10 , the optimization process for maximum thrust is repeated at a higher Mach number, $M=0.2$ (Fig. 13). Although the trend along the optimization process is similar to that of case 1 , where $M=0.1$, the maximum thrust is reached early at a relatively low plunge amplitude, $h_{o}=1.53$, and $\alpha_{o}=20 \mathrm{deg}$. The thrust value of 0.95 is about $30 \%$ smaller than that of case 1 . It appears that as the Mach number increases, large suction pressures due to leading-edge vortices cannot be sustained at plunge and pitch amplitudes as high as in case 1 . This may be attributed to the weakened and displaced leading-edge vortex due to compressibility effects.

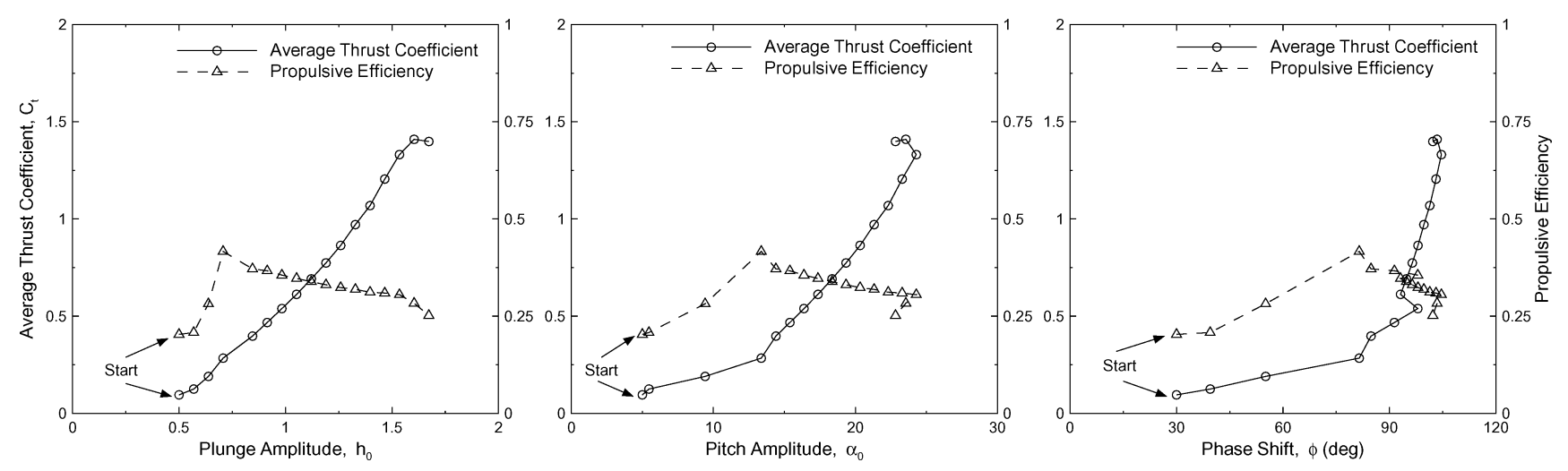

Fig. 7 Optimization steps for case $1: \boldsymbol{\beta}=\mathbf{0}$. 

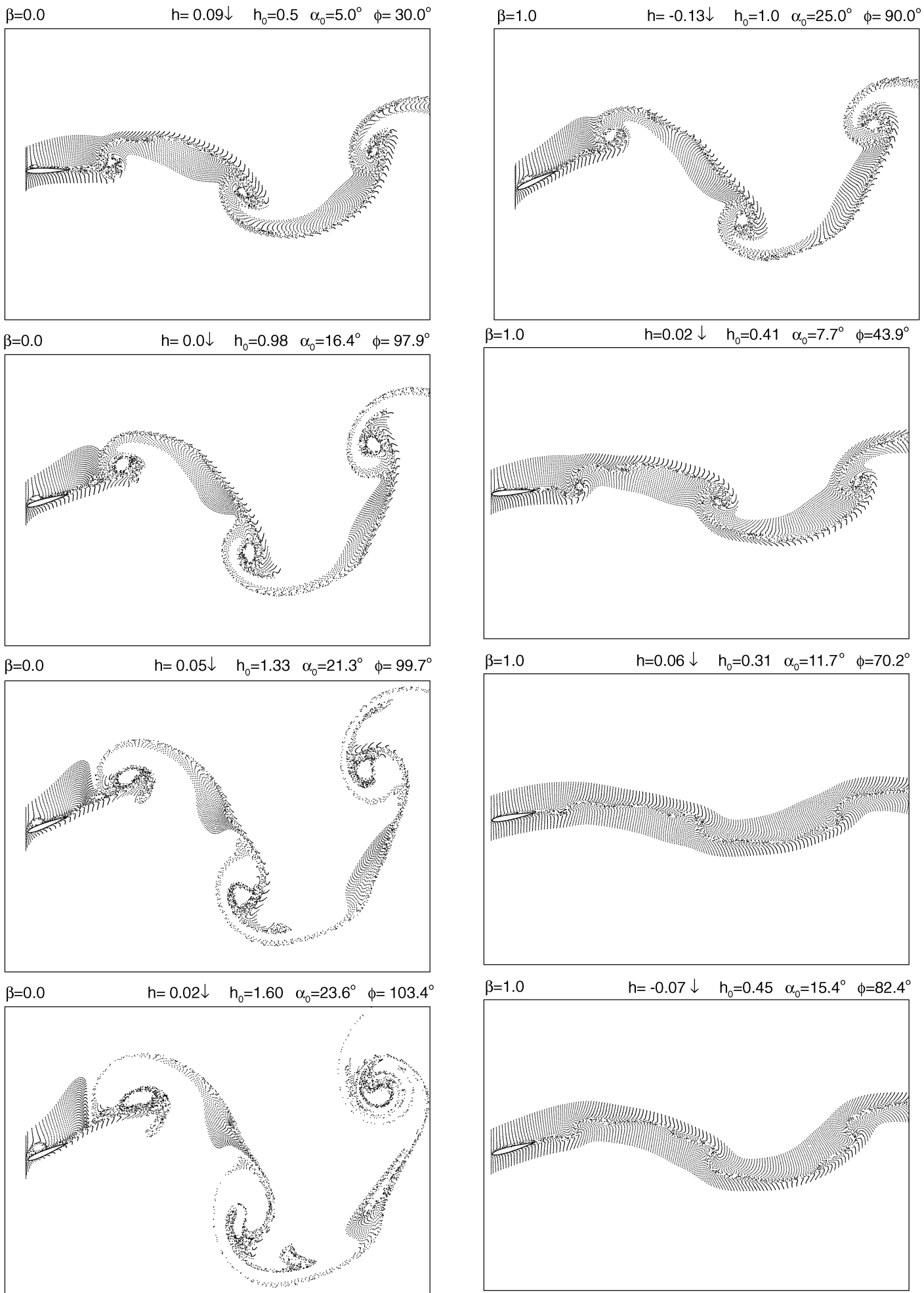

Fig. 8 Instantaneous particle traces at the instant of maximum thrust along the optimization steps for cases 1 and 3.

In case 11 , the flow is assumed to be fully turbulent. It is apparent that as the flow separation and vortex formation at the leading edge is delayed due to turbulence, higher plunge and pitch amplitudes, $h_{o}=2.11, \alpha_{o}=36.6$, and a corresponding high thrust value of $C_{t}=2.64$ may now be achieved.

The optimum flapping motions for cases $1-3$ and 9 are shown in Fig. 14. It is clearly observed that the plunge amplitude plays a significant role in thrust generation. It is also observed that to improve the propulsive efficiency, the plunge amplitude is to be reduced and the pitch amplitude is to be increased. Note that the phase shift between the plunge and the pitch motions, which is in the 85-100-deg range, helps the effective angle of attack to be reduced at the mean plunge position, where the plunge velocity is maximum.

The variation of the effective angle of attack the airfoil sees along a flapping period is given in Fig. 15 for cases $1-3$. The flapping period starts at $\theta=0 \mathrm{deg}$, which corresponds to the $h=-h_{o}$ position of the airfoil. In agreement with the earlier observation, for a high thrust production, as in cases 1 and 2, the flapping airfoil stays at large effective angles of attack for a large fraction of the flapping 

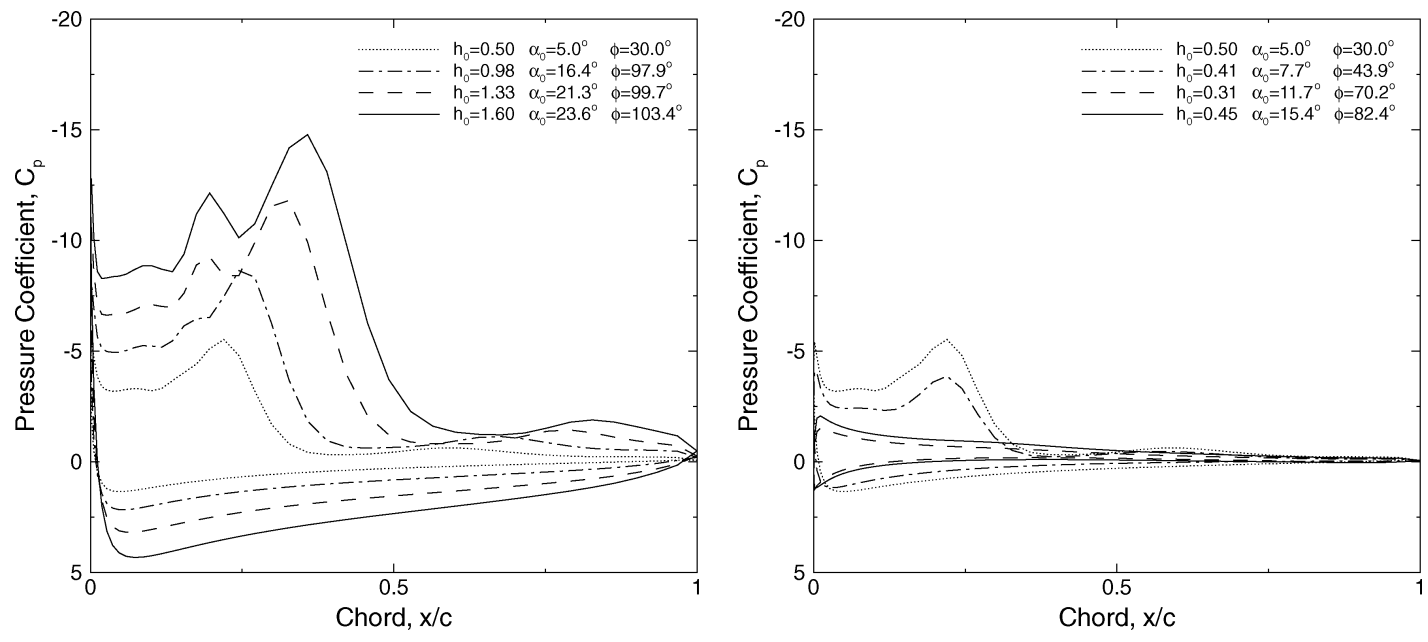

Fig. 9 Variation of surface pressure distribution along the optimization steps for cases 1 and 3: $\beta=1.0$.
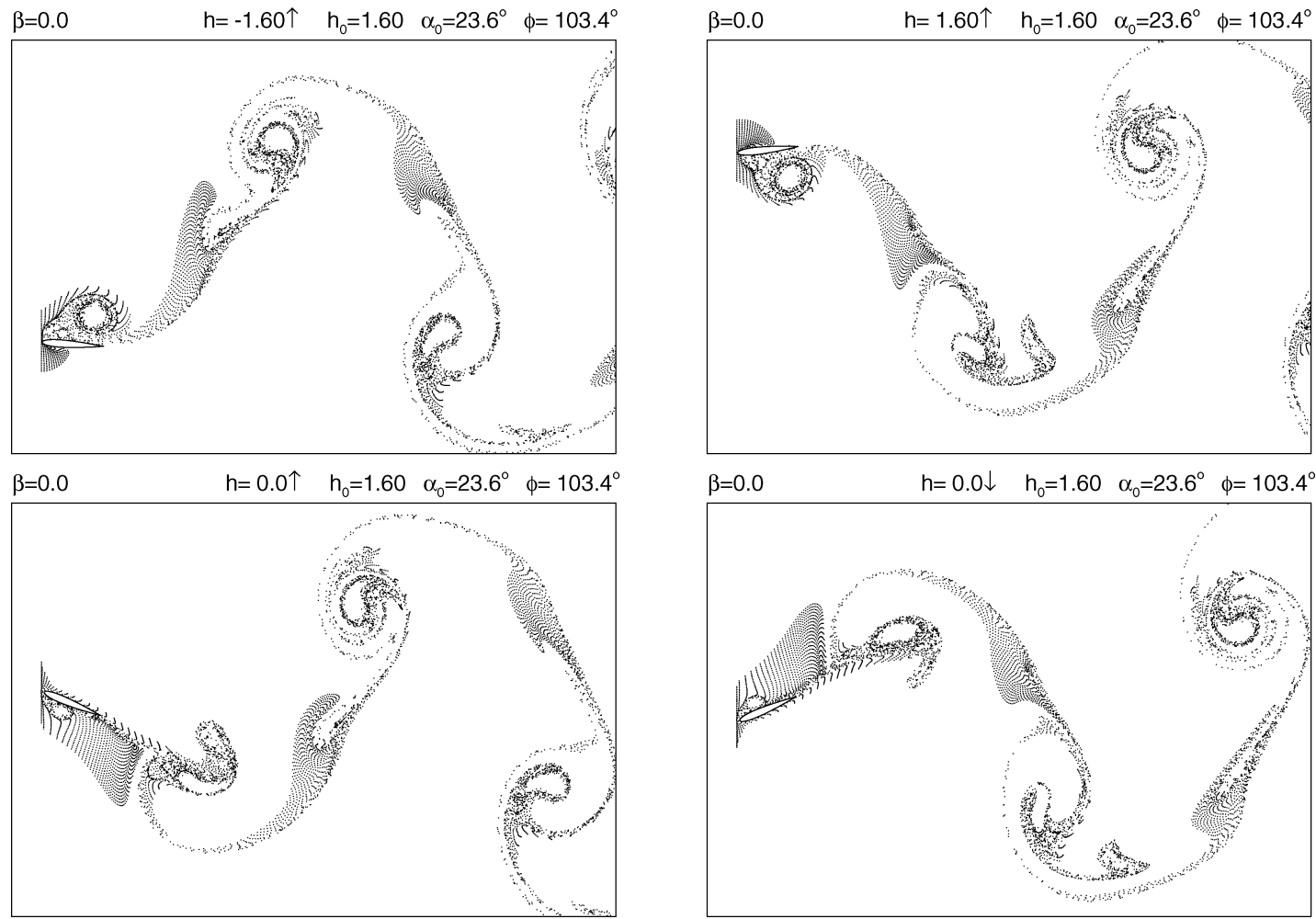

Fig. 10 Instantaneous particle traces along a period of the optimized flapping motion for case 1.
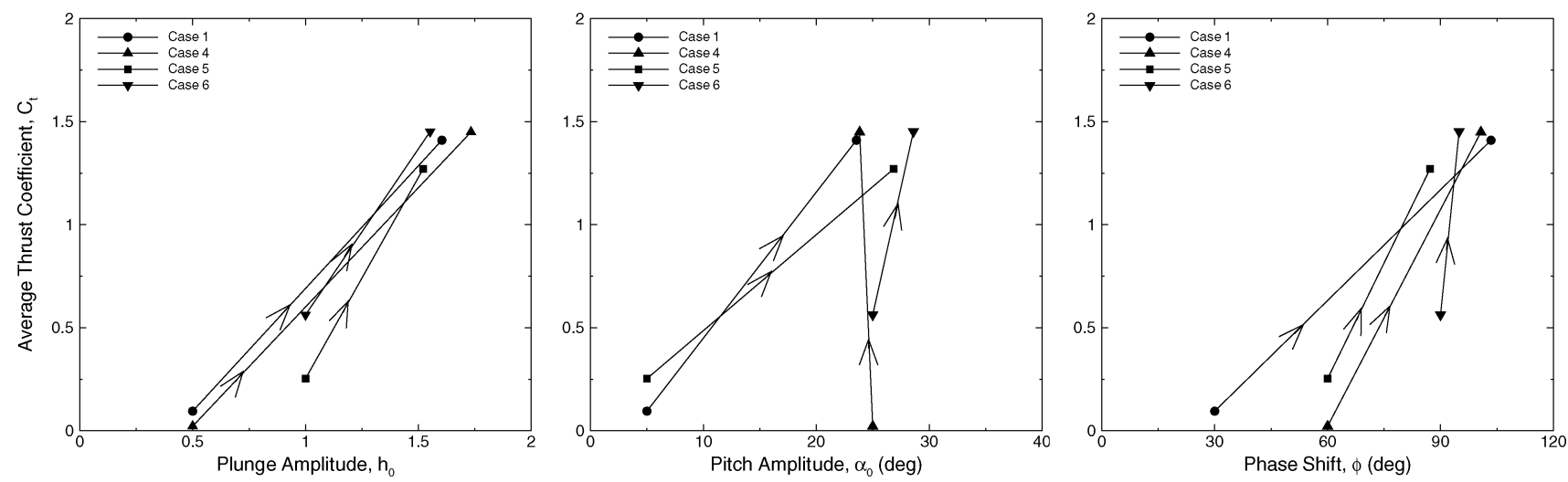

Fig. 11 Maximization of thrust coefficient $(\beta=0)$ with various starting conditions, cases 1 and 4-6. 

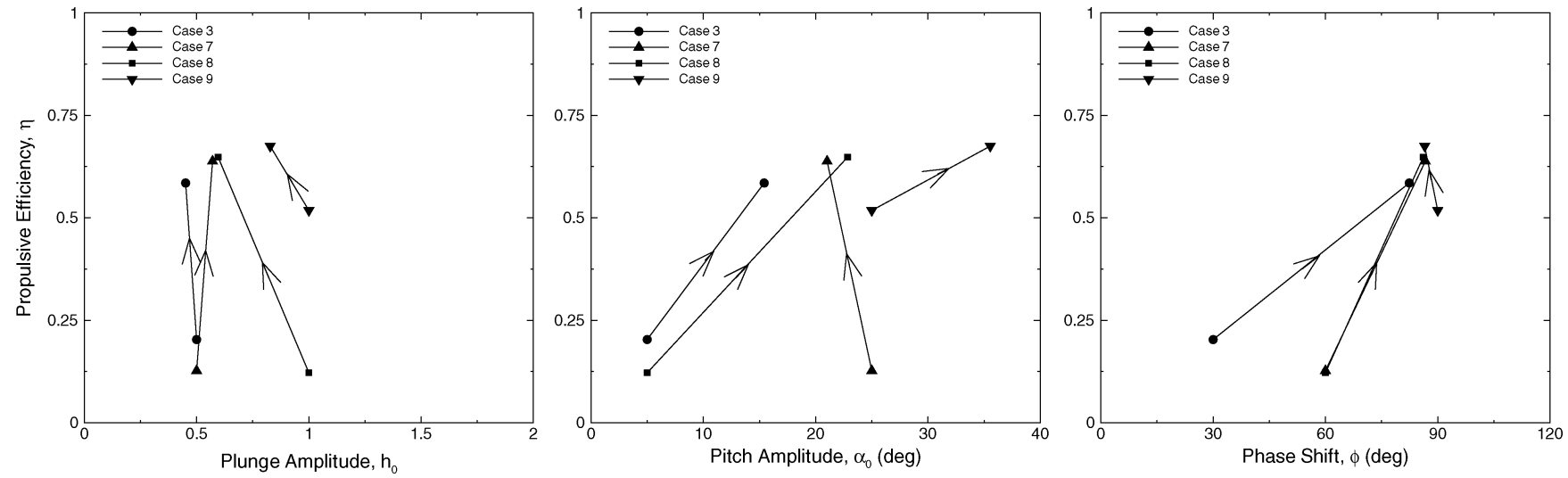

Fig. 12 Maximization of propulsive efficiency $(\beta=1)$ with various starting conditions, cases 3 and 7-9.
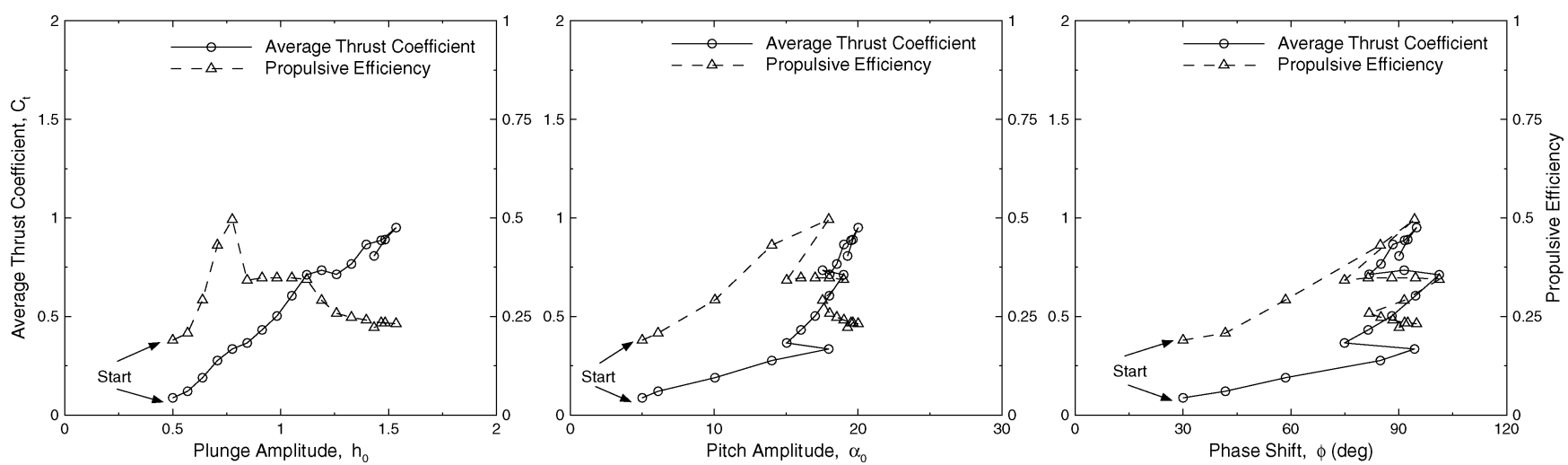

Fig. 13 Optimization steps for case 10: $\beta=0$.

Case 1

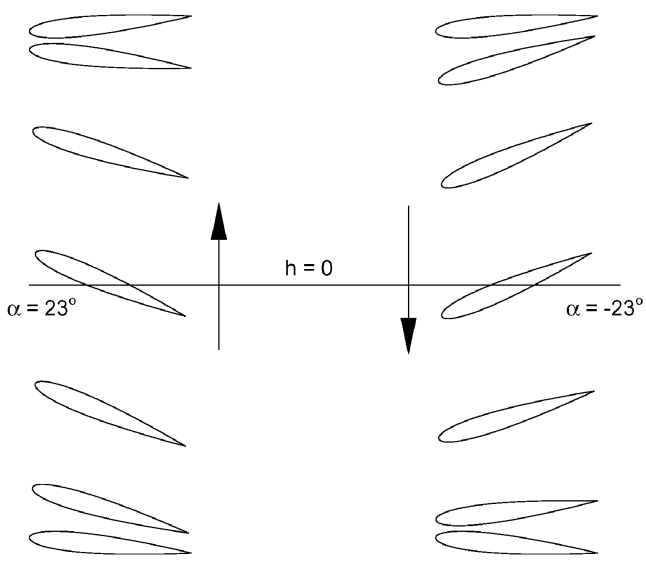

Case 3
Case 2

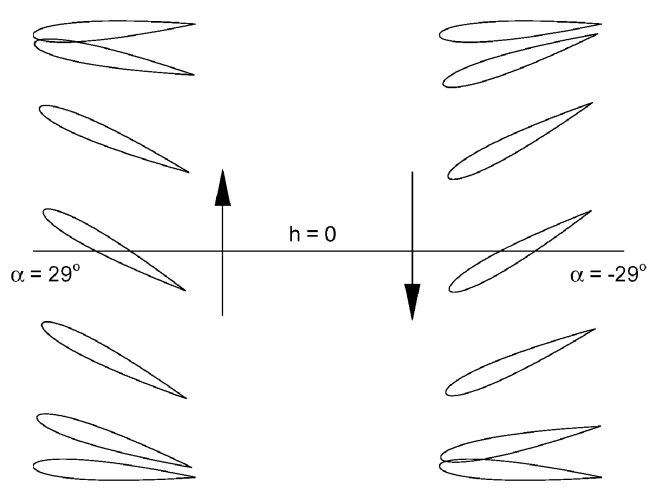

Case 9
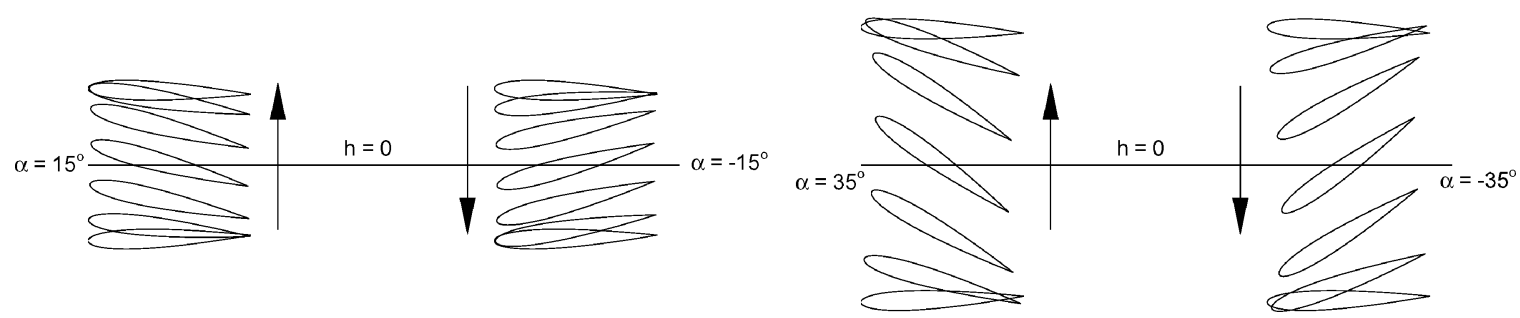

Fig. 14 Optimized flapping motions. 


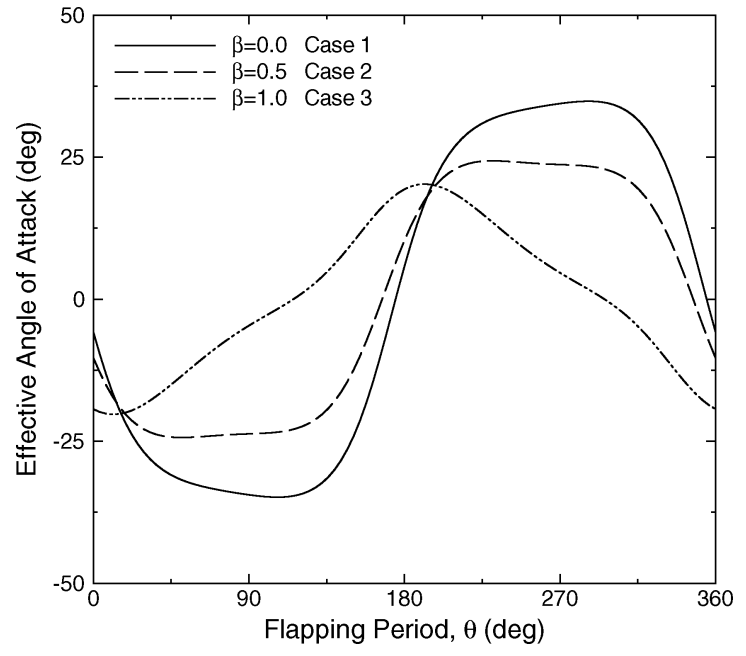

Fig. 15 Variation of effective angle of attack along a flapping period.

period. The maximum effective angle of attack occurs at about $h \approx 0$ $(\theta=90,270 \mathrm{deg})$, the mean plunge position. As a result, a strong leading-edge vortex formation is promoted. On the other hand, for an efficient flapping as in case 3, not only is the duration in which the airfoil sees large effective angles of attach reduced, but also the position of the maximum effective angle of attach shifts to $h= \pm h_{o}$ $(\theta=0,180,360 \mathrm{deg})$, the minimum and the maximum plunge amplitudes. In contrast to cases 1 and 2, the effective angle of attack at the mean plunge position is now set about $0 \mathrm{deg}$, which apparently prevents the leading-edge vortex formation.

\section{Conclusions}

A gradient-based numerical optimization is successfully applied to maximize the thrust generation and/or propulsive efficiency of an airfoil flapping in a combined plunge and pitch motion. The optimization of thrust generation and propulsive efficiency together is achieved with a weighted and normalized objective function. The parallel implementation of the optimization algorithm is shown to be quite robust. Thrust generation of a flapping airfoil is maximized at large plunge amplitudes as large leading-edge vortices form and shed into the wake. The thrust-producing airfoil stays at a large effective angle of attack during most of the flapping period. On the other hand, the propulsive efficiency of flapping airfoils may be increased by reducing the plunge amplitude and the effective angle of attack and, consequently, by preventing the formation of leading-edge vortices. Further research is in progress to implement the present optimization method to the thrust generation of flapping airfoils in a biplane configuration.

\section{Acknowledgment}

The present work was partially supported by the NATO Research and Technology Organization under Project TX01-02.

\section{References}

${ }^{1}$ Mueller, T. J. (ed.), Fixed and Flapping Wing Aerodynamics for Micro Air Vehicles, Vol. 195, Progress in Aeronautics and Astronautics, AIAA, Reston, VA, 2001.

${ }^{2}$ Shyy, W., Berg, M., and Lyungvist, D., "Flapping and Flexible Wings for Biological and Micro Air Vehicles" Progress in Aerospace Sciences, Vol. 35, No. 5, 1999 , pp. $455-505$.

${ }^{3}$ Lai, J. C. S., and Platzer, M. F., "Jet Characteristics of a Plunging Airfoil," AIAA Journal, Vol. 37, No. 12, 1999, pp. 1529-1537.

${ }^{4}$ Jones, K. D., Dohring, C. M., and Platzer, M. F., "An Experimental and Computational Investigation of the Knoller-Betz Effect," AIAA Journal, Vol. 36, No. 7, 1998, pp. 1240-1246.

${ }^{5}$ Anderson, J. M., Streitlien, K., Barrett, D. S., and Triantafyllou, M. S., "Oscillating Foils of High Propulsive Efficiency," Journal of Fluid Mechanics, Vol. 360, 1998, pp. 41-72.

${ }^{6}$ Tuncer, I. H., and Platzer, M. F., "Thrust Generation Due to Airfoil Flapping," AIAA Journal, Vol. 34, No. 2, 1995, pp. 324-331.

${ }^{7}$ Tuncer, I. H., and Platzer, M. F., "Computational Study of Flapping Airfoil Aerodynamics," Journal of Aircraft, Vol. 35, No. 4, 2000, pp. 554-560.

${ }^{8}$ Tuncer, I. H., Lai, J., Ortiz, M. A., and Platzer, M. F., "Unsteady Aerodynamics of Stationary/Flapping Airfoil Combination in Tandem," AIAA Paper 97-0659, Jan. 1997.

${ }^{9}$ Tuncer, I. H., Walz, R., and Platzer, M. F., "A Computational Study on the Dynamic Stall of a Flapping Airfoil," AIAA Paper 98-2519, June 1998.

${ }^{10}$ Isogai, K., Shinmoto, Y., and Watanabe, Y., "Effects of Dynamic Stall on Propulsive Efficiency and Thrust of a Flapping Airfoil," AIAA Journal, Vol. 37, No. 10, 2000, pp. 1145-1151.

${ }^{11}$ Isogai, K., and Shinmoto, Y., "Study on Aerodynamic Mechanism of Hovering Insects," AIAA Paper 2001-2470, June 2001.

${ }^{12}$ Jones, K. D., and Platzer, M. F., "Experimental Investigation of the Aerodynamic Characteristics of Flapping-Wing Micro Air Vehicles," AIAA Paper 2003-0418, Jan 2003.

${ }^{13}$ Jones, K. D., Bradshaw, C. J., Papadopoulos, J., and Platzer, M. F., "Improved Performance and Control of Flapping-Wing Propelled Micro Air Vehicles," AIAA Paper 2004-0399, Jan 2004.

${ }^{14}$ Jones, K. D., Duggan, S. J., and Platzer, M. F., "Flapping-Wing Propulsion for a Micro Air Vehicle," AIAA Paper 2001-0126, Jan 2001.

${ }^{15}$ Jones, K. D., Castro, B. M., Mahmoud, O., Pollard, S. J., Platzer, M. F., Neef, M. F., and Hummel, D., "A Collobirative Numerical and Experimental Investigation of Flapping-Wing Propulsion," AIAA Paper 2002-0706, Jan 2002.

${ }^{16}$ Platzer, M. F., and Jones, K. D., "The Unsteady Aerodynamics of Flapping-Foil Propellers," 9th International Symposium on Unsteady Aerodynamics, Aeroacoustics and Aeroelasticity of Turbomachines, Ecole Centrale de Lyon, Lyon, France, Sept. 2000.

${ }^{17}$ Tuncer, I. H., "A 2-D Unsteady Navier-Stokes Solution Method with Moving Overset Grids," AIAA Journal, Vol. 35, No. 3, 1997, pp. 471-476.

${ }^{18}$ Kuruvila, G., Ta' asan, S., and Salas, M. D., "Airfoil Optimization by the One-Shot Method," AGARD Rept. 803, Nov. 1994.

${ }^{19}$ Tuncer, I. H., "Parallel Computation of Multi-Passage Cascade Flows with Overset Grids," Selected Papers from Parallel Computational Fluid Dynamics Workshop, edited by U. Gulcat and D. R. Emerson, Istanbul Technical Univ., Istanbul, Turkey, 1999, pp. 81-89.

${ }^{20}$ Tuncer, I. H., and Kaya, M., "Optimization of Flapping Airfoils for Maximum Thrust," AIAA Paper 2003-0420, Jan 2003. 